\title{
Optimierung von chemisch-technologischen Systemen zur Stofftrennung
}

\author{
Dr. habil. Gennadi Wilkow
}

In der vorliegenden Arbeit wird eine Klasse von chemisch-technologischen Trennsystemen (CTTS) behandelt, in denen Standardprozesse der chemischen Technologie ablaufen: Rektifikation, Absorption, Desorption u. a. Die Systeme trennen nahezu ideale Mehrstoffgemische.

Charakteristisch fuir diese Klasse von CTTS ist ihr großer Energieverbrauch. Infolgedessen führen bereits minimale Abweichungen von optimalen Betriebsbedingungen zu wesentlichen ökonomischen Verlusten. Die Optimierung von CTTS wird sowohl bei der Projektierung (Projektierungsaufgabe optimaler CTTS, PO) als auch bei deren Steuerung (Aufgabe optimaler Steuerung, OS) durchgeführt. In der Regel werden diese Aufgaben getrennt gelöst: bei der Projektierung wird nicht beruicksichtigt, daß technologische Apparate mit automatischen Steuerungssystemen ausgeriistet werden, deren Komplexität, Leistung, und Kosten maßgeblich von statischen und dynamischen Eigenschaften der Apparate abhängt. Bei getrennter Lösung der Aufgaben für PO und OS werden häufig unterschiedliche Optimumskriterien angewendet sowie unterschiedliche Preise für Rohstoffe, Energie und Warenerzeugnisse zugrundegelegt. Im Ergebnis kann die getrennte Aufgabenlösung von PO und OS zur Effektivitätssenkung der Funktion von CTTS und zu Problemen bei der Entwicklung rationeller Steuerungssysteme fuihren. Theoretisch ist der Weg gemeinsamer Aufgabenlösung von PO und OS durch Nutzung mehrfacher Iterationsberechnung mathematischer Modelle von technologischen Apparaten der CTTS möglich. Dieser Weg stellt jedoch strenge Forderungen an die Exaktheit der mathematischen Modelle und erfordert eine leistungsfähige Hardware, wobei die optimale Lösung nicht immer garantiert ist.

In dieser Arbeit wird ein Herangehen zur gemeinsamen Aufgabenlösung von PO und OS vorgestellt, dabei liegt der Schwerpunkt nicht bei mathematischen, sondern bei technologischen Besonderheiten der betrachteten CTTS. Die Methode basiert auf Ermittlung und nachfolgender Anwendung gemeinsamer Eigenschaften (der Optimalitätsbedingungen*) optimal organisierter CTTS verschiedener Klassen.

Es wird angenommen, daß einzelne Klassen optimal organisierter CTTS, die über ausreichend komplexe Strukturen verfuigen, gemeinsame Eigenschaften haben. Es ist jedoch nicht möglich, den Grad der Komplexität, der für das Vorhandensein solcher Eigenschaften erforderlich ist, zu bestimmen. Für die Feststellung dieser Eigenschaften ist es deshalb notwendig, eine Analyse der CTTS durchzufuihren.
Eine derartige Analyse wird auf der Basis von Systemeigenschaften und Informationsstrukturen in folgender Reihenfolge durchgefuihrt:

In der ersten Stufe wird das Informationskriterium für die relative Organisiertheit der zu untersuchenden CTS ermittelt:

$$
\eta=\left(\mathrm{H}_{\text {input }}-\mathrm{H}_{\text {output }}\right) / \mathrm{H}_{\text {input }} \text {, }
$$

wobei $\mathrm{H}_{\text {input }}$ und $\mathrm{H}_{\text {output }}$ die Informationsenthropie (Maß der Ordnung) der jeweilen Ein- und Ausgangsströme der CTS ist.

Für verschiedene Klassen CTTS ist die konkrete Form des Kriteriums unterschiedlich und von Eigenschaften der zu trennenden Mehrstoffgemische abhängig. Für CTS, deren Struktur der Abb. 1 entspricht, hat das Kriterium folgendes Aussehen:

$$
\eta=1-\left(\sum_{j=1}^{n} H_{D j}+\sum_{j=1}^{n} H_{W j}\right) / \sum_{j=1}^{n} H_{F j},
$$

Für den Fall der Trennung nahezu idealer Mehrstoffgemische gilt

$$
\begin{aligned}
& H_{F j}=-F_{j} * \sum_{i=1}^{m} Z_{i j} \bullet \ln Z_{i j}, \\
& H_{D j}=-D_{j}^{*} \sum_{i=1}^{m} X_{i j}+\bullet \ln X_{i j}-, \\
& H_{W j}=-W_{j}^{*} \sum_{i=1}^{m} X_{i j}-\bullet \ln X_{i j}-,
\end{aligned}
$$

wobei

$\mathrm{F}_{\mathrm{j}}$ - den Energieverbrauch im j-ten Trennsubsystem, $D_{j}, W_{j}$ - den Verbrauch der aus dem j-ten Subsystem austretenden Komponentenströme,

$\mathrm{Z}_{\mathrm{ij}}, \mathrm{X}_{\mathrm{ij}}$ - die Konzentration der i-ten Komponente im Zulauf und in den Ausgangsströmen des j-ten Subsystems,

m - die Zahl der Komponenten des zu trennenden Gemisches,

n - die Zahl der Subsysteme darstellen.

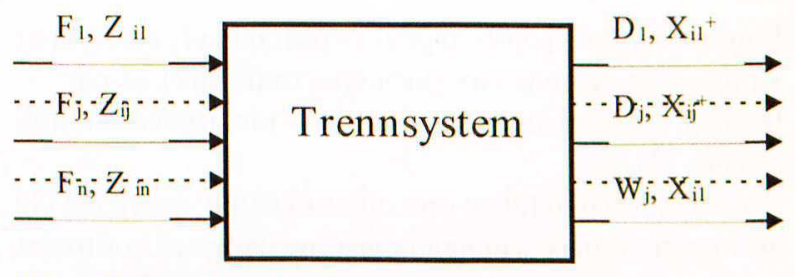

Abb. 1. Strukturschema eines Trennsystems

Das Kriterium (1) bewertet die relative Organisiertheit der CTTS. Es ist dimensionslos und kann sich von Null 
(keine Trennung - minimale Organisiertheit) bis Eins (vollständige Trennung - maximal mögliche Organisiertheit) ändern.

In der zweiten Stufe wird die Wirkung verschiedener Stellgrößen auf die Organisiertheit von CTTS, die durch das Kriterium (1) abgeschätzt werden, analysiert.

Die Änderung der Stellgrößen wirkt sich unterschiedlich auf die Organisiertheit aus. Nach dem Wirkungscharakter können sie in intensive und extensive Stellgrößen eingeteilt werden. Die intensiven Stellgrößen können die Organisiertheit von CTTS bei gleichbleibendem Energieaufwand und konstantem nichtenergetischem Aufwand erhöhen oder bei Erhöhung des Aufwandes senken. Die extensiven Stellgrößen können die Organisiertheit von CTTS nur bei einer Erhöhung von energetischem und nichtenergetischem Aufwand steigern.

Die dritte Stufe der CTTS-Analyse ist die Formulierung der Optimierungsaufgabe fuir CTTS.

Das gleiche Ziel, z. B. die Erhöhung der Trennqualität, kann entweder durch intensive oder extensive Einwirkung auf die CTTS erreicht werden. Im ersten Fall wird die Qualität der Trennung hauptsächlich durch die Einbeziehung zusätzlicher Informationen in den technologischen Prozeß (Kenntnis über den zweckmäßigen Einsatz energetischer und nichtenergetischer Ressource) erreicht. Im zweiten Fall wird sie durch die Erhöhung des genannten Aufwandes erreicht.

Bei der Optimierung sollte der extensive Weg nur dann gewählt werden, wenn alle Möglichkeiten intensiver Einwirkung ausgeschöpft sind.

Die mathematische Formulierung der Aufgabe ist also folgende: nach den intensiven Stellgrößen ( $U^{\text {int }}$ ) soll das Kriterium (1) das Maximum erreichen, nach den extensiven Stellgrößen (U $\left.{ }^{\text {ext }}\right)$ sollte sein Wert möglichst minimiert werden. Die Gleichungen der Materialbilanz und vorgegebene Anforderungen zur Qualität der Produktfraktion $\left(X_{i j}><X_{i j}{ }^{v o r g}\right)$ sind dabei einzuhalten.

$$
\begin{aligned}
& \min _{U^{\text {ext }}} \max _{U^{i n t}} \eta\left(U^{e x t}, U^{i n t}, F_{j}, Z_{i j}, X_{i j}{ }^{+}, X_{i j}-\right), \\
& D_{j}^{*} X_{i j}{ }^{+}+W_{j}^{*} X_{i j}-=F_{j}^{*} Z_{i j}, \\
& F_{j}=D_{j}+W_{j}, \\
& \sum_{j=1}^{m} X_{i j}=1 \\
& X_{i j}><X_{i j}{ }^{\operatorname{vorg}} .
\end{aligned}
$$

In dieser Formulierung stellt das Kriterium (1) die Kennzahl für energetischen (nichtenergetischen) Aufwand dar.

Die vierte Stufe der Analyse ist die Lösung der Optimierungsaufgabe.

Lösungen, die bei der Optimierung des Kriteriums (1) nach den intensiven Stellgrößen erzielt wurden, ermöglichen es, die maximal mögliche Trennung bei einem konstanten energetischen (nichtenergetischen) Aufwand in der CTS zu erreichen. Diese Lösungen stehen zu keiner ökonomisch begründeten Optimierungsaufgabe im Widerspruch. Dies bedeutet, daß sie bei der Lösung von Aufgaben sowohl bei der Projektierung optimaler CTS als auch bei optimaler Steuerung angewandt werden miissen. Die Nutzung genannter Bedingungen ermöglicht eine gemeinsame Aufgabenlösung für die Optimierung bei der Projektierung CTS und in der Steuerung.

Die Analyse der Ausdriicke (1) und (2) zeigt, daß sie nicht nur für eine CTTS sondern für eine ganze Klasse von CTTS zutreffen, die durch ein konkretes Optimalitätskriterium charakterisiert wird. Das bedeutet, daß die optimale Lösung in einer allgemeinen Form die Optimumsbedingungen für eine ganze Klasse CTTS darstellt. Die ermittelten Optimumsbedingungen widerspiegeln die gemeinsamen Eigenschaften optimaler CTTS einer bestimmten Klasse.

In der mathematischen Formulierung der Optimierungsaufgabe (2) sind physikalische Größen enthalten, die die Ein- und Ausgangsströme von CTTS charakterisieren. In diesem Fall enthält auch die Lösung dieser Aufgabe nur die genannten physikalischen Größen. Diese Schlußfolgerung erlaubt es, die Lösung der Gesamtoptimierungsaufgabe in zwei Stufen durchzufuihren.

In der ersten Stufe werden die Austrittsgrößen der CTTS $\left(D_{j}, W_{j}, x_{i j}{ }^{+}, x_{i j}{ }^{-}\right)$ermittelt, die als Ausgangsdaten für die Optimierung jedes einzelnen Trennapparats (Dekomposition komplexer von CTTS in optimale Subsysteme) erforderlich sind. Dabei wird das Optimalitätskriterium (1) angewendet.

Die zweite Stufe beinhaltet die Optimierung der einzelnen Trennapparate mit Anwendung ökonomischer Optimalitätskriterien. Bei der Lösung dieser Aufgabe muß gewährleistet werden, daß die Bedingungen der Dekomposition unabhängig von den für einzelne Apparate angewendeten Optimumskriterien erfuillt sind. Damit wird eine systemimmanente Widerspruchsfreiheit für die CTTS und die einzelnen Trennapparate erreicht.

Der ersten Stufe geht die Ermittlung des besten Verfahrens für die Kopplung der einzelnen Trennapparate in der CTTS voraus, die zweite Stufe wird mit der Ausarbeitung des optimalen Steuerungssystems für die CTTS abgeschlossen.

In einzelnen Fällen wird es nicht möglich sein, die Stellgrößen der CTTS in intensive und extensive einzuteilen. Das bedeutet, daß die zu untersuchende CTTS eine Komplexität besitzt, die für eine Formulierung gemeinsamer Eigenschaften nicht ausreicht. Die Komplexität ist in diesem Fall zu erhöhen (z. B. durch eine Anbindung der in der technologischen Kette folgenden Apparate), und die Analyse muß für die komplexere CTTS durchgefuihrt werden.

Das in dieser Arbeit vorgeschlagene Herangehen erlaubt es, die komplizierte Aufgabe der CTTS-Optimierung durch eine Reihe einfacherer Aufgaben zu ersetzen und die Ermittlung quantitativer Ergebnisse wesentlich zu vereinfachen.

\section{Verfasser}

\section{Dr. habil. Gennadi Wilkow}

Staatliche Technische Universität Jaroslawl Fachbereich für Technische Kybernetik

Tel. 007 (0) 852 / 446623 